\title{
Comparison of Multivariate Analysis Methodologies in a Palliative Care Setting
}

\author{
Isabel Pinto Doria ${ }^{1}$, Ana Sousa Ferreira ${ }^{2}$, Otília Dias ${ }^{3}$, \\ Helena Bacelar-Nicolau ${ }^{4}$, Georges Le Calvé ${ }^{5}$
}

\author{
${ }^{l}$ Universidade de Lisboa, FP-UL, LEAD and CEAUL Portugal, irpdoria@fp.ul.pt \\ ${ }^{2}$ Universidade de Lisboa, FP-UL, LEAD and UNIDE, CEAUL, Portugal, asferreira@fp.ul.pt \\ ${ }^{3}$ IPS, ESTBarreiro and CEAUL, Portugal, otilia.dias@estbarreiro.ips.pt \\ ${ }^{4}$ Universidade de Lisboa, FP-UL, LEAD and CEAUL, Portugal, hbacelar@fp.ul.pt \\ ${ }^{5}$ Société Française de Statistique, g.lecalve@free.fr
}

\begin{abstract}
SUMMARY
This study is focused on measuring the quality and the satisfaction with the palliative care provided to oncology patients in domicile. The SERVQUAL methodology adapted for the Portuguese context was used to evaluate the quality of palliative care and patient satisfaction. The Portuguese SERVQUAL questionnaire is composed of five perception scales and two questionnaires, one about the patient and another about the caregiver. The data analysis presented is the analysis of the answers to the five perception scales, composed of partial ordered variables, evaluating different aspects of quality and satisfaction. The data was analysed comparing metric and symbolic approaches, using Principal Component Analysis Methods and Agglomerative Hierarchical Cluster Analysis Models. The results suggest that a symbolic approach provides a more comprehensive analysis for this kind of data.
\end{abstract}

Key words: Agglomerative Hierarchical Cluster Analysis, Multivariate Analysis, Palliative Care, Principal Component Analysis, Symbolic variables.

\section{Introduction}

In 1990, the World Health Organization defined palliative medicine as "The active total care of patients whose disease is not responsive to curative treatment. Control of pain, other symptoms and psychological, social and spiritual problems, is paramount. The goal of palliative care is achievement of the best quality of life for patients and their families." 
In Portugal the document "Health in Portugal - A strategy for the turn of the Century", presented by the Ministry of Health in 1999, stated as one of its objectives the humanization of palliative care through alternative forms of assistance to traditional hospitalization. Domiciliary care in the context of teams of health professionals and trained caregivers is one of those alternatives.

In this context, a multidisciplinary team developed a Project of Humanization of Palliative Care in the Domicile Context, whose primary objective was to measure the quality and the satisfaction with the palliative care provided to oncology patients in domicile and to train volunteers for benevolent care. This research was carried out under the aforementioned project.

Fifty-eight caregivers of patients from the Service of Communitarian Medicine of the Francisco Gentil Portuguese Oncology Institute (40\%) and Odivelas Centre of Healthcare (60\%) answered a Portuguese SERVQUAL questionnaire. This questionnaire is composed of five perception scales (Block I), a questionnaire about the patient and a questionnaire about the caregiver.

The results presented here are an analysis of the answers to the five dimensions (perception scales) of Block I of the Portuguese SERVQUAL questionnaire, evaluating different aspects of quality and satisfaction:

A. Tangibles ( 7 items) - Physical facilities, equipment.

B. Reliability (5 items) - Ability to perform the promised service accurately.

C. Responsiveness (8 items) - Willingness to help users and provide a prompt service.

D. Assurance (9 items) - Inspiring credibility and trust.

E. Empathy (11 items) - Personal attention.

Block I is a multiple-scale with 40 items, scored from 1 to 5 ("Strongly disagree" to "Strongly agree") plus 6 ("Not applicable") and 9 ("Don't know/No answer"). These items are therefore measured on a scale with partly ordered modalities.

Two approaches are used to analyse this data - a metric one, and a symbolic one based on the coefficient $\mathrm{s}_{\mathrm{LC}}$ (Le Calvé, 1977; Doria, 2008) - and their results are compared. 
In both approaches Principal Component Analysis (PCA) and Agglomerative Hierarchical Cluster Analysis (AHCA) are used. As is known, PCA makes it possible to reduce dimensionality with minimum loss of information contained in the data and AHCA permits classification of the items:

- PCA analysis of the 58 caregivers' answers on the five perception scales will give us the principal components that explain patient satisfaction and quality of palliative care;

- AHCA will give us a typology for patient satisfaction and for the quality of the palliative care provided by a team of experts (doctor/nurse).

This study is meant to continue the work reported in previous papers (Bacelar-Nicolau et al., 2005; Doria et al., 2007).

\section{Data Characterization}

Although the instrument used to measure the quality and satisfaction of palliative care in domicile is a questionnaire, since it is answered by caregivers of patients who are severely or terminally ill (often cancer patients and sometimes deceased), it was concluded that it would be applied in the form of an interview, because the questions involve feelings and emotions, and sometimes it was so difficult to conduct the interview that the session had to be interrupted and resumed on another day.

The caregivers' responding to our study are mostly female (78\% female and $22 \%$ male) with mean age $59 \pm 15$ (mean \pm SD) years; their age distribution is presented in Figure 1. The relation of caregiver to patient is most frequently husband/wife (44\%), followed by daughter/son (35\%), other $(16 \%)$, and mother/father (5\%).

The patients' gender distribution is very similar (52\% male and $48 \%$ female), and the mean age is $69 \pm 12$ years with an almost symmetric distribution of age (Figure 2). 


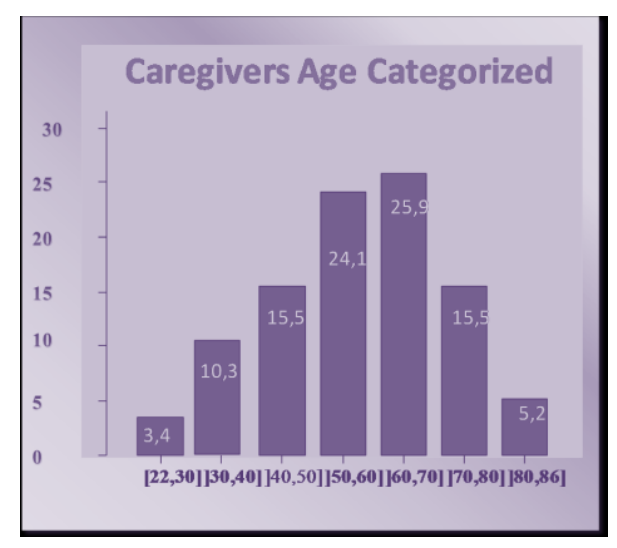

Figure 1. Categorized caregivers' age distribution

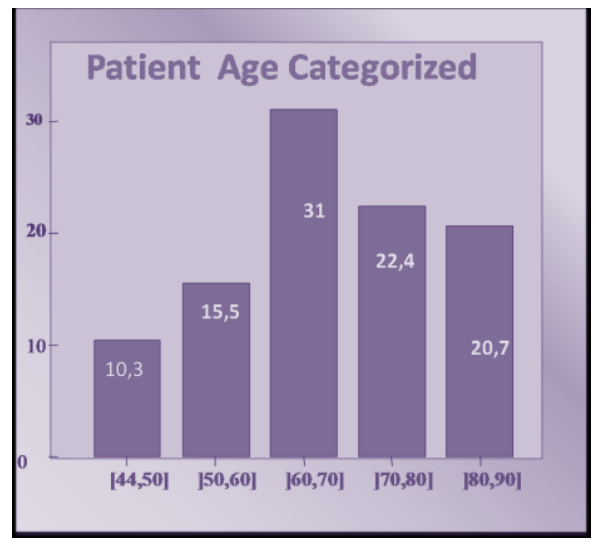

Figure 2. Categorized patients' age distribution

\section{Methods}

The five perception scales, codified as metric variables, were compared using the methodologies from Principal Component Analysis (PCA) and Agglomerative Hierarchical Cluster Analysis (AHCA), directly applied to the similarity matrix $R_{P}$ Pearson Correlation. Several classical aggregation criteria were combined (Single Linkage (SL), Complete Linkage (CL), Average Linkage (AL)) with the Pearson Correlation Coefficient.

These scales, codified as symbolic/complex modal variables, were compared using the methodologies from Principal Component Analysis (PCA) and Agglomerative Hierarchical Cluster Analysis (AHCA) directly applied to the generalized similarity matrix $S_{L C}$ (e.g. Doria, 2008).

The values of the $s_{L C}$ coefficient were combined with five aggregation criteria, two of which are classical: Single Linkage (SL) and Complete Linkage (CL), and three of which are probabilistic: AVL, AV1 and AVB (BacelarNicolau, 1988; Nicolau and Bacelar-Nicolau, 1998).

The results obtained with the two approaches are compared. 


\subsection{The metric approach}

The answers given by the 58 palliative caregivers for each of the perception scales were codified as percentages, since each scale comprises a different number of items. Their descriptive distribution is presented in the following table (Table 1):

Table 1. Descriptive statistics (Mean, SD) of the perception scales.

\begin{tabular}{lll}
\hline Perception scales & Mean & SD \\
\hline Tangibles (\%) & 66.31 & 17.64 \\
Reliability (\%) & 88.83 & 10.13 \\
Responsiveness (\%) & 91.08 & 11.65 \\
Assurance (\%) & 77.93 & 22.95 \\
Empathy (\%) & 89.09 & 12.43 \\
\hline
\end{tabular}

These data were analysed using PCA, an exploratory method of multivariate analysis.

Principal components are linear combinations of the original variables which permit reduction of dimensionality with minimum loss of data variability, to obtain a better understating of the phenomenon under study.

ACHA is a set of multivariate analysis methods that aims to obtain a hierarchy of partitions of a set of objects or variables. An ACHA algorithm has three steps:

- Calculation of a coefficient of comparison (similarity or dissimilarity), $\gamma(\mathrm{x}, \mathrm{y})$, between pairs of elements of the set to be classified;

- Calculation of a coefficient of comparison (aggregation criteria), $Г(A, B)$, between pairs of classes of the set to be classified;

- Hierarchy of classifications (Dendrogram).

\subsection{The symbolic approach}

The answers given by the fifty eight palliative caregivers to each of the scales were codified as symbolic modal variables, which resulted in a three- 
dimensional symbolic data matrix $M(58 \times 5 \times 7)$, in which each individual is described according to the profile obtained from the answers to the item modalities of each of the five scales (Table 2).

Table 2. Extract from the $\mathrm{M}(58,5,7)$ three-dimensional data matrix, scale A

\begin{tabular}{cccccccc}
\hline \multirow{2}{*}{ Caregivers } & \multicolumn{7}{c}{ Tangibles } \\
\cline { 2 - 8 } & 1 & 2 & 3 & 4 & 5 & 6 & 9 \\
\hline 1 & 0.000 & 0.286 & 0.143 & 0.143 & 0.143 & 0.286 & 0.000 \\
2 & 0.000 & 0.143 & 0.000 & 0.429 & 0.143 & 0.429 & 0.000 \\
$\ldots$ & & & & & & & \\
58 & 0.000 & 0.000 & 0.143 & 0.000 & 0.857 & 0.000 & 0.000 \\
\hline
\end{tabular}

As we know, in a symbolic data matrix, lines correspond to symbolic objects (SOs) whereas columns correspond to symbolic variables, which may take values such as subsets of categories, intervals of real axes, or frequency distributions. Its cells can thus contain not just one value, as usual, but several values which can be weighted and linked by logical rules and taxonomies (Bock and Diday, 2000).

Formally, a modal variable $Y$, with domain $\mathrm{Y}$, defined in a set $E=\{a, b, \ldots\}$ of objects, is a mapping $Y(a)=\left(U(a), \pi_{a}\right), a \in E$, where $\pi_{a}$ is a non-negative measure in Y, generally a frequency distribution (absolute or relative) or a probability or weight distribution on the domain $\mathrm{Y}$ of possible observed values, and $U(a) \subseteq \mathrm{Y}$ is the support for $\pi_{a}$ in domain Y (Bock and Diday, 2000). In this study's data matrix (Table 1) the entries correspond to the relative frequency distributions.

\subsubsection{The generalized similarity coefficient $s_{L C}$}

The similarity coefficients between variables $\mathrm{s}, \mathrm{s}_{\mathrm{LC}}$ and $\mathrm{P}_{\mathrm{L}}$ were inspired by an idea originally from Daniels (1944), later developed by Lerman (1973) and generalized by Le Calvé (1977). In this approach, each variable is associated with a score matrix, whose definition depends on the nature of the initial variable, as well as on the nature of the variable with which it is to be compared. The basic coefficient, $s$, is defined as the scalar product between the score 
matrices, the $\mathrm{s}_{\mathrm{LC}}$ coefficient is the standardized coefficient $s$, under a certain reference hypothesis, and the $\mathrm{P}_{\mathrm{L}}$ coefficient corresponds to the probabilistic coefficient. Recently, coefficients $\mathrm{s}, \mathrm{S}_{\mathrm{LC}}$ and $\mathrm{P}_{\mathrm{L}}$ were generalized to the comparison of symbolic modal variables, among other symbolic variables (Doria et al., 2007; Doria, 2008).

Definition of the $S, S_{L C}$ and $P_{L}$ coefficients (Le Calvé, 1977): A probabilistic similarity coefficient between variables $\mathrm{X}, \mathrm{Y}$, designated by $\mathrm{P}_{\mathrm{L}}$, is defined by the probability of $\mathrm{S}_{\mathrm{LC}}$ being smaller than

$$
\mathrm{s}_{\mathrm{LC}}: \mathrm{P}_{\mathrm{L}}(\mathrm{x}, \mathrm{y})=\mathrm{P}\left(\mathrm{S}_{\mathrm{LC}} \leq \mathrm{sLC}(\mathrm{x}, \mathrm{y})\right)=\Phi(\mathrm{sLC}(\mathrm{x}, \mathrm{y})) .
$$

The random variable $\mathrm{S}_{\mathrm{LC}}$ is the standardised similarity,

$$
\begin{aligned}
& \mathrm{S}_{\mathrm{LC}}=\left(\mathrm{S}_{\mathrm{X}, \mathrm{Y}}(\mathrm{w})-\mu\right) / \sigma, \\
& \mathrm{S}_{\mathrm{X}, \mathrm{Y}}\left(\theta, \theta^{\prime}\right)=<\theta \mathrm{X} \theta^{\mathrm{t}}, \theta^{\prime} \mathrm{Y} \theta^{, \mathrm{t}}>, \forall \mathrm{w} \in\left(\theta, \theta^{\prime}\right),
\end{aligned}
$$

considering the set of all permutation couples,

$$
\Omega=\Theta(\mathrm{I}) \mathrm{x} \Theta(\mathrm{I})
$$

defined on I, provided with a uniformly distributed probability measure. $\mathrm{P}_{\mathrm{L}}$ is the probability distribution function of that standarised similarity being observed. Under very general conditions on the score matrices, the random variable $\mathrm{S}_{\mathrm{LC}}$ has asymptotic standard normal distribution, the coefficients $\mathrm{S}_{\mathrm{LC}}$ and $\mathrm{s}$ are the actual values of the random variables $\mathrm{S}_{\mathrm{LC}}$ and $\mathrm{S}$, respectively, and $\Phi$ denotes the standard normal distribution function.

Let us consider a three-way data matrix $\mathrm{M}=\left[\left(x_{i(j) 1}, x_{i(j) 2}, \ldots, x_{i(j) m}\right)\right]$ $(i=1, \ldots, N$ entities; $j=1, \ldots, p$ modal variables, all of which with the same number $m$ of modalities), in which $x_{i(j) k}(i=1, \ldots, N ; k=1, \ldots, m)$ is the value taken by the modal variable $\underline{X}_{j}$ for statistical unity $i$ - in this case, a relative frequency distribution.

Definition of the score (Doria, 2008): The score of the modal variable $\underline{X}_{j}$ is defined as follows 


$$
\begin{aligned}
& x_{i i^{\prime}}=\operatorname{aff}\left(i, i^{\prime}\right), \text { if } \quad i \neq i^{\prime} \\
& x_{i i}=0
\end{aligned}
$$

where $\operatorname{aff}\left(i, i^{\prime}\right)=\sum_{k=1}^{m} \sqrt{x_{i(j) k} x_{i^{\prime}(j) k}}$

indicates the affinity coefficient (e.g., Bacelar-Nicolau, 2000) between the profiles (relative frequencies) from the answers given by individuals $i$ and $i$.

\section{Results}

\subsection{Results obtained with the metric approach}

PCA and AHCA were applied to the 58 responses of caregivers to the five perception scales of the Portuguese SERVQUAL questionnaire.

The results of PCA are summarized in Table 3 and Figures 3,4 and 5.

\begin{tabular}{|c|c|c|c|c|c|}
\hline & \multicolumn{5}{|c|}{ Components } \\
\hline & 1 & 2 & 3 & 4 & 5 \\
\hline Eigenvalues & 2.571 & 1.028 & 0.6457 & 0.449 & 0.3068 \\
\hline$\%$ Total variance & 51.422 & 20.556 & 12.907 & 8.987 & 6.128 \\
\hline Tangibles (\%) & & :765: & -.072 & .289 & .195 \\
\hline Reliability (\%) & |.695 &.$- \ddot{142}$ & $\because 687$ & .135 & -.078 \\
\hline Responsiveness (\%) & | .792 | & -.422 & -.114 & -.174 & .390 \\
\hline Assurance $(\%)$ & $\mathbf{I} .778$ I & .358 & -.075 & -.459 & -.223 \\
\hline Empathy $(\%)$ & $! .53^{\prime}$ & -.340 & -.386 & .326 & -.246 \\
\hline
\end{tabular}

Table 3. Principal Component Analysis results

It can be seen that the first principal component explains $51.4 \%$ of the total data variability, and is a general component. The second component is attached to the Tangibles scale - physical facilities, equipment, and is responsible for $20.6 \%$ of the variability. The $(1,3)$ and $(2,3)$ planes reveal a third component close to the Reliability scale - ability to perform the treatments accurately. 


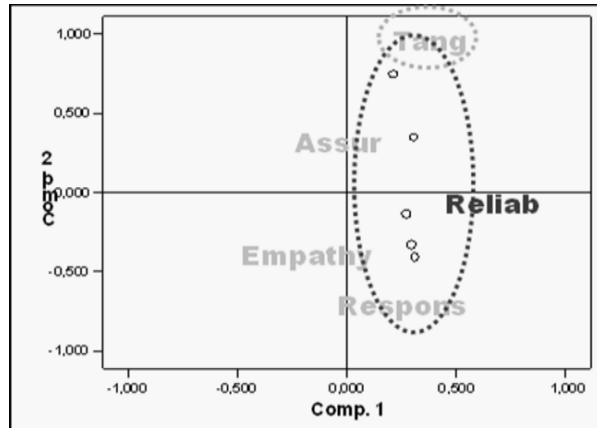

Figure 3. Representation of the five perception scales from the Portuguese SERVQUAL questionnaire in the factorial plane $(1,2)$ obtained from PCA

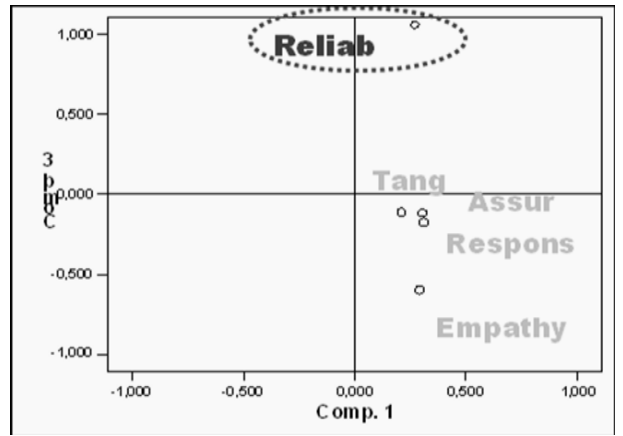

Figure 4. Representation of the five perception scales from the Portuguese SERVQUAL questionnaire in the factorial plane $(1,3)$ obtained from PCA

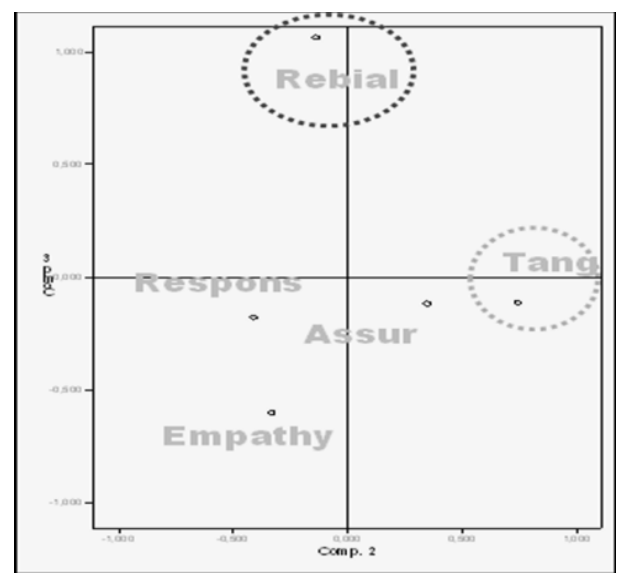

Figure 5. Representation of the five perception scales from the Portuguese SERVQUAL questionnaire in the factorial plane $(2,3)$ obtained from PCA

The best result obtained from the AHCA $\left(r_{P}+\right.$ Single Linkage $)$ model is given by the partition into three clusters (Figure 6):

Cluster $1=\{\mathrm{C}$. Responsiveness, E. Empathy $\}$ Cluster which refers to prompt conduct of services and inspiring of sympathies in patients, in which there is a higher number of answers "4-Agreement" and "5-Total agreement"; 
Cluster $2=\{$ B. Reliability $\}$ Cluster that refers to inspiring of credibility and trust, in which there is a higher number of answers "4-Agreement" and "5-Total agreement";

Cluster $3=\{$ A. Tangibles, D. Assurance $\}$ Cluster which refers to equipment and assurance;

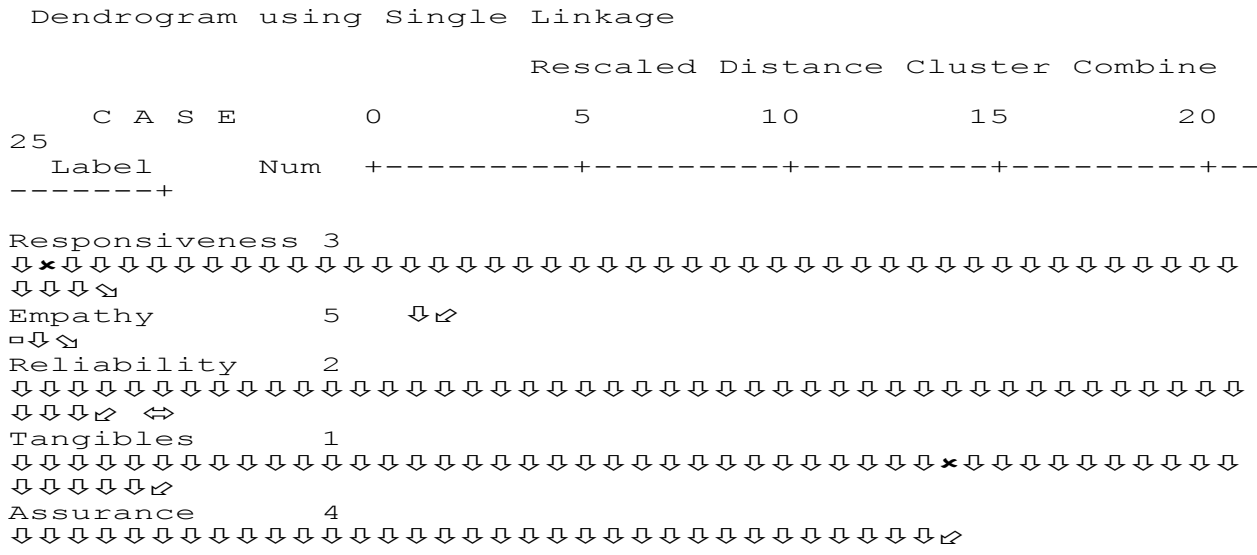

Figure 6. Dendrogram obtained from the AHCA ( $r_{P}+$ Single Linkage) model. Level 2 is the most important one, according to the SPSS "level statistics" criterion

\subsection{Results obtained with the generalized similarity coefficient sLC}

Agglomerative Hierarchical Cluster Analysis (Figure 7) and Principal Component Analysis (Table 4, Figure 8) are used on the similarity matrix obtained with the coefficient $s_{L C}$.

The best result obtained from the AHCA $\left(s_{L C}+\right.$ Single Linkage) model is given by the partition into two clusters (Figure 7):

Cluster $1=\{$ A. Tangible Elements $\}$ Cluster which refers to the equipment and has a different answering profile from all the others - the answers reveal the lower satisfaction of caregivers;

Cluster $2=\{$ B. Reliability, E. Empathy, C. Responsiveness, D. Assurance $\}$ 
Table 4. Principal Component Analysis of $S_{L C}$ matrix results

\begin{tabular}{|c|c|c|c|c|c|}
\hline & \multicolumn{5}{|c|}{ Components } \\
\hline & 1 & 2 & 3 & 4 & 5 \\
\hline Eigenvalues & 28.22 & 15.26 & 11.67 & 7.14 & 4.48 \\
\hline$\%$ Total variance & 42.27 & 22.85 & 17.48 & 10.69 & 6.71 \\
\hline Tangibles (\%) & 2.160 & -3 & -.627 & .072 & .161 \\
\hline Reliability (\%) & 1.925 & 1.002 & & -.880 & -1.001 \\
\hline Responsiveness (\%) & 2.194 & .668 & .289 & 2.242 & -.631 \\
\hline Assurance (\%) & 3.205 & 0.461 & 2.224 & 1.146 & -.254 \\
\hline Empathy (\%) & & 1.214 & & .134 & 1.730 \\
\hline
\end{tabular}

Cluster which refers to the ability to flawlessly apply treatments, to pay personal attention to patients, to promptly conduct the services and to inspire credibility and trust, in which there is a higher number of answers "4-Agreement" and "5-Total agreement".

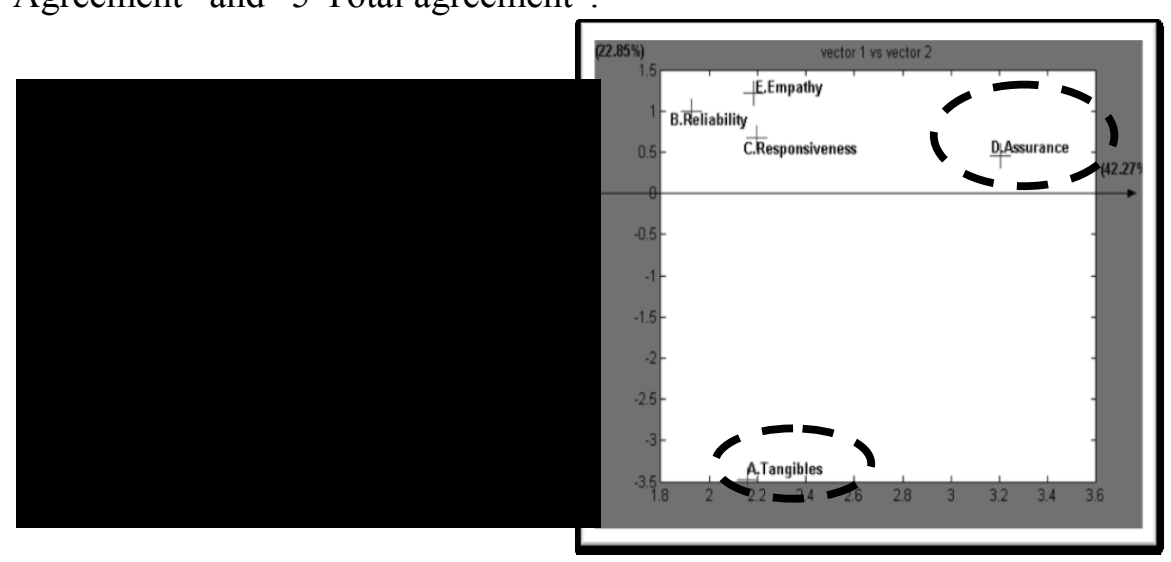

Figure 7. Dendrogram obtained from the AHCA $\left(s_{L C}+\right.$ Single Linkage) model. Level 3 is the most important one, followed by level 2, according to the "statistics of levels" (Lerman, 1970; Bacelar-Nicolau, 1972)
Figure 8. Representation of the five perception scales from the Portuguese SERVQUAL questionnaire in the factorial plane $(1,2)$, obtained from PCA on the $S_{L C}$ matrix 
In PCA directly applied to the similarity matrix $S_{L C}$, it can be seen that the first principal component explains $42.27 \%$ of the total data variability. In it, the scale "D. Assurance", that is, the capability of inspiring credibility and trust, stands out (Figure 8), having a larger proportion of "5-Total agreement" and "9-Don't know/No answer".

Cluster 2 refers to the ability to perform the treatments accurately ( $3^{\text {rd }}$ component), to provide personal attention, to provide a prompt service and to inspire credibility and trust $\left(1^{\text {st }}, 3^{\text {rd }}\right.$ components). We recognize the $1^{\text {st }}$ factorial dimension in this partition (Figure 7, Figure 8, Table 4).

\section{Conclusions}

Data analysis revealed that caregivers are less satisfied with the palliative care provided to their patients as far as equipment is concerned, but they strongly recognized the accurate performance of treatments and personal attention that inspire credibility and trust.

These data show a very strong hierarchical classificatory structure for both analyses; the same dendrograms were obtained with different aggregation criteria.

The AHCA results are different in the two analyses, but the PCA results are similar in both.

There is more coherence between the $\mathrm{s}_{\mathrm{LC}}$ results produced by PCA and AHCA.

It is advantageous to use the $\mathrm{s}_{\mathrm{LC}}$ coefficient, as it permits the use of complete data (including "not applicable" and "don't know/no answer") without losing information.

\section{REFERENCES}

BaBakus E., Mangold W.G. (1992): Adapting the SERVQUAL Scale to Hospital Services: An Empirical Investigation. Health Services Research 26(6): 767-786. 
Nicolau F., Bacelar-Nicolau H. (1998): Some Trends in the Classification of Variables. In: C.Hayashi, N. Ohsumi, K. Yajima, Y. Tanaka, H. H. Bock, Y. Baba (eds.): Data Science, Classification, and Related Methods, Springer-Verlag: 89-98.

Le Calvé G. (1977): Un indice de similarité pour des variables de type quelconque. Revue Statistique et Analyse des Données 01/02: 39-47.

Daniels H.E. (1944): The relation between measures of correlation in the universe of sample permutations. Biometrika, 33, 129-135.

Bock H.-H.; Diday E. (Eds.) (2000): Analysis of Symbolic Data: Exploratory Methods for Extracting Statistical Information from Complex Data. Series: Studies in Classification, Data Analysis, and Knowledge Organization, Springer.

Bacelar-Nicolau H. (1972): Analyse d'un Algorithme de Classification. Thèse de 3 ème cycle, Université Pierre et Marie Curie, Paris.

Bacelar-Nicolau H. (1980). Contribuições ao Estudo dos Coeficientes de Comparação em Análise Classificatória. Phd Thesis, Faculdade de Ciências da Universidade de Lisboa.

Bacelar-Nicolau H. (1988): Two Probabilistic Models for Classification of Variables in Frequency Tables. In: Bock, H.H.(eds.): Classification and Related Methods of Data Analysis, 181-186. North Holland.

Bacelar-Nicolau H. (2000): The affinity coefficient. In: H.-H. Bock and E. Diday (eds.): Analysis of Symbolic Data: Exploratory Methods for Extracting Statistical Information from Complex Data. Springer-Verlag, Berlin/Heidelber: 160-165.

Bacelar-Nicolau H., Dias O., Sousa Ferreira A. (2005): Humanização dos Cuidados Paliativos: Análise Multivariada de um Questionário de Qualidade/Satisfação ao Apoio Domiciliário. In: Livro de Resumos das XII Jornadas de Classificação e Análise de Dados, JOCLAD 2005: 70-71.

Doria I., Dias O., Sousa Ferreira A., Le Calvé G., Bacelar-Nicolau H. (2007): Comparison of methodologies of multivariate analysis in a palliative care context. In: Livro de resumos da XI Conferencia Española de Biometria e Primer Encuentro Iberoamericano de Biometria (CEIB2007): 101-102.

Doria I. (2008): Representações euclidianas de dados: Uma abordagem para variáveis heterogéneas. Phd Thesis, Universidade de Lisboa, Lisboa.

Lerman I.C. (1970): Sur l'Analyse des Données Préalable à une Classification Automatique. Proposition d'une Nouvelle Mesure de Similarité. MSH, rapport 32 (8e. année), Paris.

Lerman I.C. (1973): Étude Distributionnelle de Statistiques de Proximité entre Structures Algébriques Finies de Même Type. Application à la Classification Automatique. Cahiers du Bureau Universitaire de Recherche Opérationnelle, n. 19. Institut de Statistique de l'Université de Paris, Paris. 